\title{
Clinical investigation into the initial diagnosis and treatment of 539 patients with stage IV lung cancer
}

This article was published in the following Dove Press journal:

OncoTargets and Therapy

31 January 2017

Number of times this article has been viewed

\section{Qian Shao \\ Shanshan Liu \\ Wei Wang \\ Yingjie Zhang \\ Fengxiang Li \\ Jianbin Li}

Department of Radiation Oncology, Shandong Cancer Hospital Affiliated to Shandong University, Shandong Academy of Medical Sciences, Jinan, People's Republic of China
Correspondence: Qian Shao; Jianbin Li

Department of Radiation Oncology (Chest Section), Shandong Cancer Hospital Affiliated to Shandong University, Shandong Academy of Medical Sciences, Road Jiyan 440, Jinan 250I I7, People's Republic of China

Tel +8653 I 67626132

Fax +8653 | 67626130

Email shaoqian2009@sina.com; lijianbin@msn.com
Objective: The aim of the study was to analyze clinical data, including the types of pathologic classification, metastatic organs, treatment strategy, and prognosis of patients with stage IV lung cancer.

Methods: A retrospective analysis of the clinical features of 539 patients with stage IV lung cancer who were initially diagnosed and treated in 2009 was conducted. There were 146 cases of single organ metastases and 393 cases of multiple organ metastases. The Kaplan-Meier method and multivariate Cox regression analysis were performed to analyze the influence of age, pathological classification, metastatic organs, and treatment strategy on overall survival.

Results: The 1-, 2-, and 3-year survival rates were 64.2\% $(n=346), 19.7 \%(n=106)$, and 1.5\% $(\mathrm{n}=8)$, respectively. Metastases to the liver and pleura predicted poor prognosis, although bone metastases predicted relatively good prognosis. The prognosis of single brain metastasis was relatively better than that of multiple brain metastases. Multi-factor analysis showed that the patient's age, different metastatic organs, the numbers of metastatic organs, and different treatment were independent risk factors for survival.

Conclusion: The prognosis for patients with stage IV lung cancer is poor. Patient's age, the type and number of metastatic organs, and method of treatment are the main factors affecting survival.

Keywords: carcinoma/lung, diagnosis, therapy, prognosis, clinical analysis

\section{Introduction}

Lung cancer has the highest morbidity and mortality rate of all malignant tumors, and is the most commonly occurring malignancy. ${ }^{1-4}$ Because there are no obvious or specific symptoms or signs at early stages, the initial diagnosis and treatment of patients at early stages accounted for only $11.2 \% .^{1}$ Although physical examinations can identify lung cancer as soon as symptoms appear, lung cancer is, generally, diagnosed in the advanced stages, at which point prognosis is poor and the likelihood of a cure is lost. The 5-year survival rate from lung cancer is reportedly $<15 \% .{ }^{4}$ This study analyzed the clinical characteristics and survival-related factors of 539 patients with stage IV lung cancer diagnosed and treated for the first time in our hospital from 2009-01-01 to 2009-12-31.

\section{Materials and methods}

\section{Inclusion criteria}

1) Primary lung lesions were diagnosed by pathology and/or cytology, 2) clinical examinations were comprehensive, including brain magnetic resonance imaging (MRI) or computed tomography (CT), enhanced chest and abdomen CT scan, systemic radionuclide bone imaging (if there were abnormal radionuclide concentration foci, 
MRI, CT, or X-ray examination was added), or systemic Positron emission tomography-CT to make the extent of the tumor clear, and clinical staging according to the seventh edition of tumor staging guidelines formulated by the American Joint Committee on Cancer, ${ }^{5} 3$ ) first diagnosed and treated (or untreated) in our hospital, without treatment in another hospital, and, 4) cases of reexamination were excluded. Written informed consent was obtained from all patients, and the institutional research ethics board of Shandong Cancer Hospital approved this study (No SDTHEC201503048).

\section{General clinical data}

Data were collected from 539 patients with stage IV lung cancer who were admitted to our hospital from 2009-01-01 to 2009-12-31. Patients with no pathologic or cytologic diagnosis and incomplete data were excluded. The median duration of patients' delay to see a doctor was as follows: 1) the shortest delay was 3 days, 2) the longest delay was 24 months, 3 ) the average delay was 2.36 months, and 4) the median delay was 2 months. Table 1 displays the clinical data.

\section{Treatment methods}

The main treatment methods were chemotherapy, radiotherapy, surgical treatment, targeted treatment, and combination therapy. There were 275 cases $(51.0 \%)$ adopting radiotherapy and chemotherapy, 117 cases $(21.7 \%)$ receiving chemotherapy, and 70 cases $(13.0 \%)$ with no treatment (Table 2 ).

\section{Follow-up}

Up to 2012-12-31, 79 patients were lost to follow-up. The follow-up rate was $85.3 \%$. The follow-up time was 1-38 months. The mean follow-up time was 16.1 months and the median follow-up time was 16 months. The follow-up of surviving patients is ongoing.

\section{Statistical methods}

Statistical analysis was performed using SPSS software (version 17; SPSS, Inc., Chicago, IL, USA). Student's $t$-test was utilized to compare the means of two samples. The $\chi^{2}$ test was used for all other comparisons. Univariate survival analysis was conducted using Kaplan-Meier analysis and the log-rank test. The significance level was $\alpha=0.05$.

\section{Results}

\section{Survival situation}

Up to 2012-12-31, the follow-up rate was $85.3 \%(460 / 539)$. The 1, 2, and 3-year survival rates were $64.2 \%(n=346)$, $19.7 \%(n=106)$, and $1.5 \%(n=8)$, respectively.
Table I Patient clinical information

\begin{tabular}{|c|c|}
\hline Parameters & Patients, n (\%) \\
\hline \multicolumn{2}{|l|}{ Sex } \\
\hline Male & $35 I(65.1)$ \\
\hline Female & $188(34.9)$ \\
\hline \multicolumn{2}{|l|}{ Age (years) } \\
\hline Range & 19-88 \\
\hline Mean age & 58.51 \\
\hline Male & 58.59 \\
\hline Female & 57.66 \\
\hline Median age & 59 \\
\hline Male & 59 \\
\hline Female & 58 \\
\hline \multicolumn{2}{|l|}{ Dividing line } \\
\hline$<60$ years & $282(52.3)$ \\
\hline$>60$ years & $257(47.7)$ \\
\hline Pathology or cytology & 539 \\
\hline Adenocarcinoma & 377 (69.9) \\
\hline Squamous carcinoma & $95(17.6)$ \\
\hline Small cell carcinoma & $50(9.3)$ \\
\hline Large cell carcinoma & $9(1.7)$ \\
\hline Adenosquamous carcinoma & $5(0.9)$ \\
\hline Carcinoid tumor & $2(0.4)$ \\
\hline Sarcomatoid carcinoma & $\mathrm{I}(0.2)$ \\
\hline \multicolumn{2}{|l|}{ Clinical symptoms } \\
\hline Cough & $43(8.0)$ \\
\hline Chest pain & $58(10.8)$ \\
\hline Dyspnea & $98(18.2)$ \\
\hline Cough, sputum & $112(20.8)$ \\
\hline Cough, blood in phlegm & $19(3.5)$ \\
\hline Cough, sputum, chest tightness & $4(0.7)$ \\
\hline Cough, sputum, chest pain & $7(1.3)$ \\
\hline Cough, sputum, dyspnea & $22(4.1)$ \\
\hline Cough, sputum, fever & $4(0.7)$ \\
\hline outside of the lung & $20(3.7)$ \\
\hline Symptoms occurring outside of the lung & $139(25.8)$ \\
\hline Headache and ostalgia & $13(2.4)$ \\
\hline Single organ metastasis & 393 \\
\hline Pleura & $112(28.5)$ \\
\hline Brain & $100(25.4)$ \\
\hline Bone & $78(19.8)$ \\
\hline Lung & $60(15.3)$ \\
\hline Liver & $29(7.4)$ \\
\hline Adrenal gland & $14(3.6)$ \\
\hline
\end{tabular}

\section{Comparison of survival of patients with single organ metastasis}

Survival for the 393 patients with different single organ metastases is as follows: pleura $(n=112 ; 28.5 \%)$, brain $(\mathrm{n}=100 ; 25.4 \%)$, bone $(\mathrm{n}=78 ; 19.8 \%)$, lung $(\mathrm{n}=60 ; 15.3 \%)$, liver $(n=29 ; 7.4 \%)$, and adrenal metastasis $(n=14 ; 3.6 \%)$. Among single organ metastasis, the survival time of patients with different metastatic organs was significantly different $(P<0.01)$. The survival time for patients with bone metastasis 
Table 2 Treatment methods

\begin{tabular}{ll}
\hline Treatment method & Patients, $\mathbf{n}$ (\%) \\
\hline Chemotherapy & $117(21.7)$ \\
Radiotherapy & $11(2.0)$ \\
Chemoradiotherapy & $275(51.0)$ \\
Chemoradiotherapy + targeted therapy & $19(3.5)$ \\
Chemotherapy + targeted therapy & $26(4.8)$ \\
Radiotherapy + targeted therapy & $4(0.7)$ \\
Surgery + chemotherapy & $3(0.6)$ \\
Surgery + chemoradiotherapy & $9(1.7)$ \\
Targeted therapy & $5(0.9)$ \\
Untreated & $70(13.0)$ \\
\hline
\end{tabular}

was relatively longer, but that of patients with liver metastasis was shorter. The survival times of patients with single organ metastasis is shown in Figure 1.

\section{Comparison of survival time in patients with single organ metastasis and multiple organ metastases}

Among the 539 patients, 393 (72.9\%) had single organ metastasis and 146 (27.1\%) had multiple organ metastases. The median survival times in single organ metastasis, two organ metastases, and three or more organs metastases were 18,15 , and 8 months, respectively. The survival time of patients with single organ metastasis, two organ metastases, and three or more organs metastases was significantly different $(P<0.01$; Figure 2).

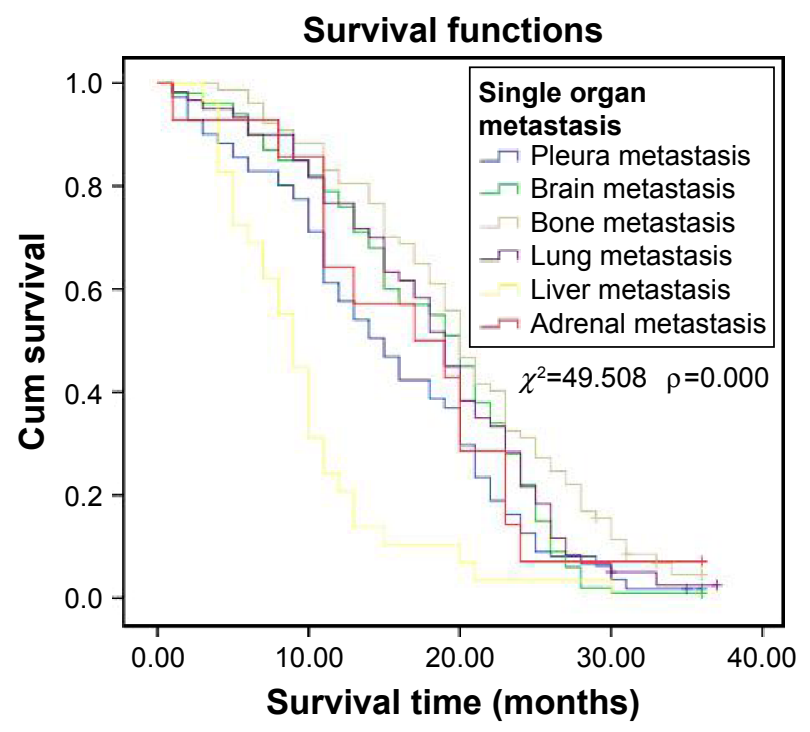

Figure I Cumulative survival time of patients with different single organ metastases.

Notes: The survival time of patients with different metastatic organs was significantly different $(P<0.01)$, with bone metastasis relatively longer, but liver metastasis shorter.

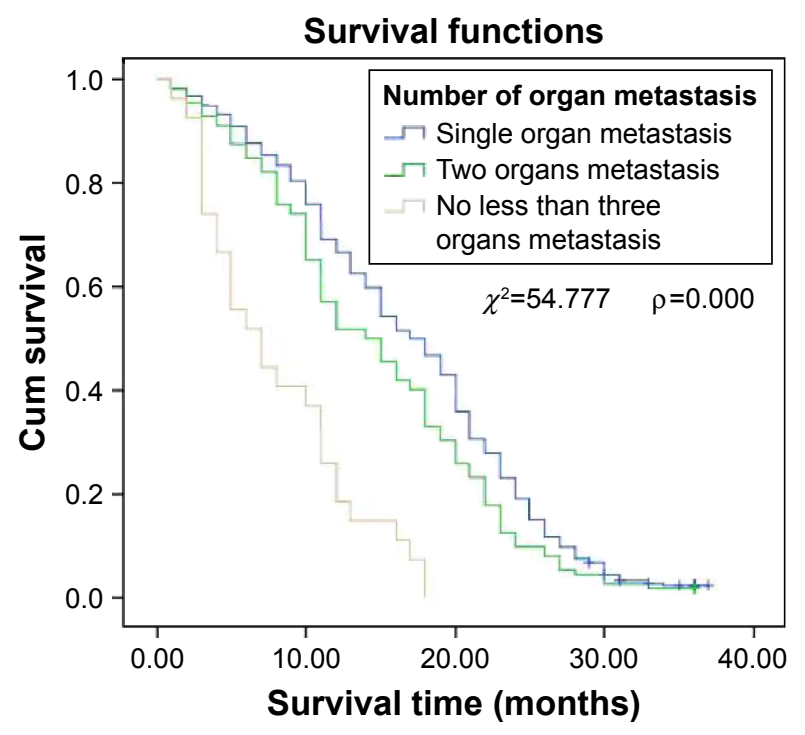

Figure 2 Cumulative survival of patients with single organ metastasis and multiple organ metastases.

Notes: The survival time of patients with single organ metastasis, two organ metastases, and three or more organ metastases were significantly different $(P<0.01)$, and single organ metastasis was better than two or more organ metastases.

\section{Comparison of survival time in patients} with single brain metastasis and patients with two or more brain metastases

Among the 393 patients with single organ metastasis, $100(25.4 \%)$ had brain metastases; 35 (35.0\%) had single brain metastasis, whereas $65(64.0 \%)$ had two or more brain metastases. Survival time was significantly better in patients with single brain metastasis than in patients with multiple brain metastases $(P<0.05$; Figure 3$)$.

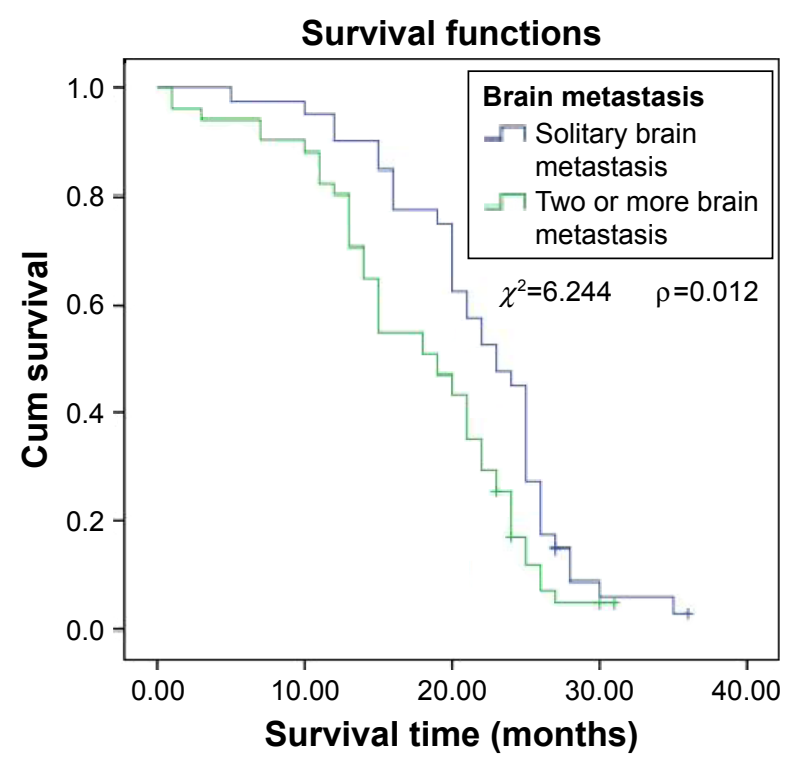

Figure 3 Cumulative survival of patients with solitary and multiple brain metastases. Notes: The survival time of patients with solitary brain metastasis was better than multiple brain metastases $(P<0.05)$. 
Relationship between radiation dose and survival time in patients with brain metastases

There was a significant difference in the survival time of patients with different radiotherapy doses. A positive correlation between radiation dose and survival time was observed. As the radiation dose varied from $<50$ to $50-59$ Gy to $\geq 60 \mathrm{~Gy}$, the survival time increased accordingly. There were significant differences between the three subgroups (the radiation dose of brain metastases $<50$ Gy, 50-59 Gy and $\geq 60$ Gy). The relationship between radiation dose and survival in patients with brain metastases is shown in Figure 4.

\section{Relationship between treatment methods} and survival

Treatment methods, such as simple chemotherapy, radiotherapy, surgery, and targeted therapy, were significantly related to survival. There were significant differences in survival as treatment method varied. Chemoradiotherapy combined with targeted therapy showed better therapeutic effects. The prognosis of patients treated with chemotherapy alone or without treatment was poor. The different therapy influenced survival time of patients $(P<0.01)$. The effect of treatment methods on survival is shown in Figure 5.

\section{Comparison of survival time in treated and untreated patients}

In patients with advanced lung cancer, survival time was significantly prolonged by receiving chemoradiotherapy and

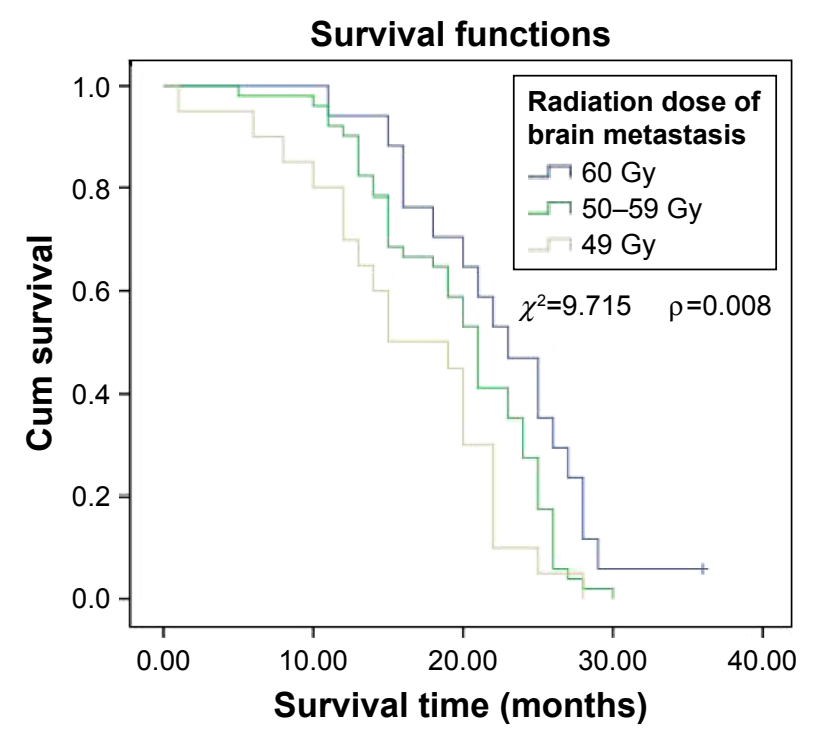

Figure 4 Relationship between radiation dose and survival in patients with brain metastases.

Notes: The radiation dose influenced survival time of patients, and the dose $\geq 60 \mathrm{~Gy}$ was better than 50-59 Gy or $<50$ Gy $(P<0.01)$.

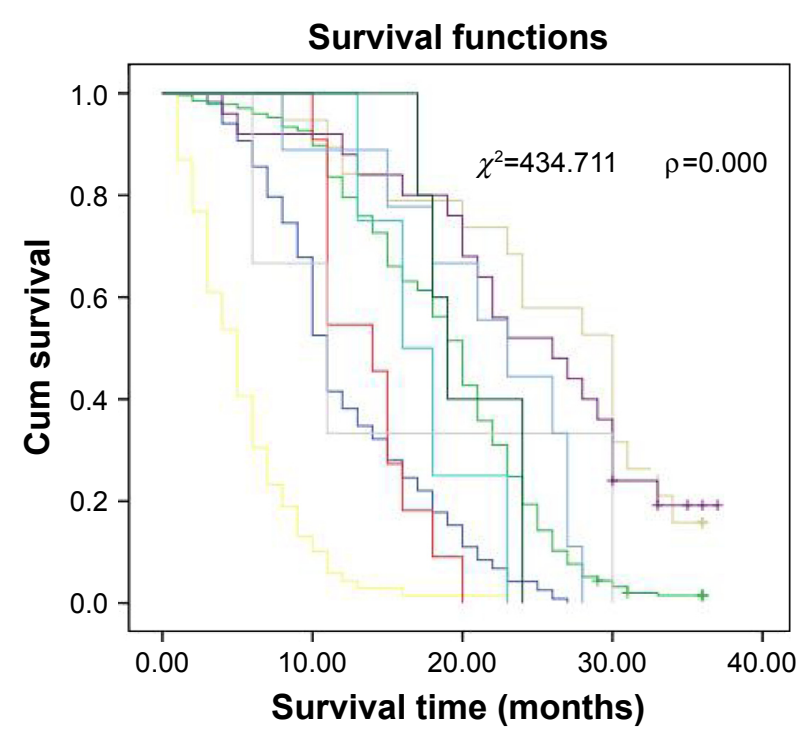

\begin{tabular}{|lc|}
\hline Treatment methods & \\
$\neg$ Chemotherapy & $-\neg$ Chemoradiotherapy \\
Chemoradiotherapy + & $-\neg$ Chemotherapy + \\
targeted therapy & targeted therapy \\
Untreated & $-\neg$ Radiotherapy \\
$\neg$ Radiotherapy + & Surgery + \\
targeted therapy & chemotherapy \\
- Surgery + & Targeted therapy \\
chemoradiotherapy & \\
\hline
\end{tabular}

Figure 5 Effects of different treatment methods on survival.

Notes: The different therapy influenced survival time of patients, and the comprehensive therapy was better than others $(P<0.0$ I $)$.

targeted therapy. The median survival time of the untreated patients was 5 months, whereas that of the comprehensively treated patients was 18 months. The survival time of patients received therapy was significantly longer than patients not received therapy $(P<0.01)$. Figure 6 shows survival times in treated and untreated patients.

\section{Multivariate correlation analysis related to survival}

Multivariate Cox regression analysis showed that age, different metastatic organs, the numbers of metastatic organs, and different treatment methods were correlated with survival time (Table 3).

\section{Discussion}

The incidence of lung cancer has shown a declining trend in developed countries, but is on the rise in developing countries. Furthermore, lung cancer is no longer a disease of old age, but has increased incidence in younger individuals. In this study, patients' ages ranged from 19 to 88 years, with a median age of 59 years. There were 282 cases $(52.3 \%)<60$ years old, and 257 cases $(47.7 \%)>60$ years old, 


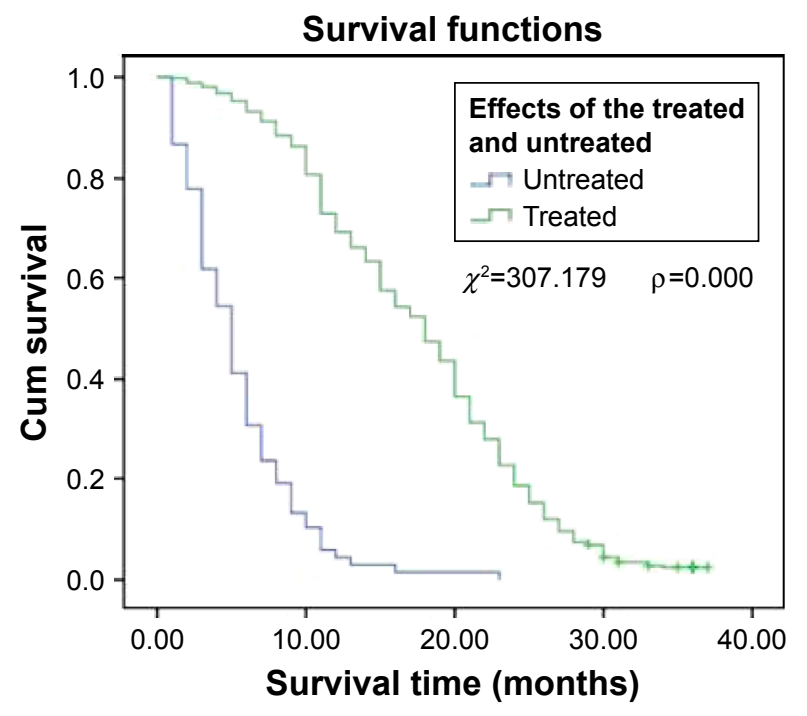

Figure 6 Comparison of survival times between treated and untreated patients. Notes: The survival time of patients who received therapy was significantly longer than in patients that did not receive therapy $(P<0.0 \mathrm{I})$.

suggesting that younger age has become another feature of lung cancer.

The occurrence of lung cancer was not only related to smoking or environmental pollution, but also to stress, bad habits, and lack of exercise. ${ }^{6-9}$ We suggest slowing down the pace of life, learning to self-decompress, developing good habits, and regularly exercising daily or weekly, because exercise can reduce the risk of lung cancer. ${ }^{10}$ People $>40$ years should have at least one physical examination each year. For those who are at high risk, a low-dose CT scan has been suggested. ${ }^{1-13}$ Early discovery, diagnosis, and treatment could be assured by the abovementioned means to improve the survival rate and reduce the mortality of lung cancer.

Early stage lung cancer does not have obvious symptoms, whereas advanced patients seldom had few symptoms. In this study, 13 patients $(2.4 \%)$ were asymptomatic, and most had pulmonary and/or extrapulmonary symptoms. The primary reported symptoms were pulmonary: 311 cases $(57.7 \%)$ had cough, sputum, chest pain, chest tightness, and dyspnea, and 19 cases $(3.5 \%)$ had sputum with blood. Extrapulmonary

Table 3 Multivariate correlation analysis related to survival

\begin{tabular}{lllll}
\hline Factors & $\begin{array}{l}\text { Regression } \\
\text { coefficient }(\boldsymbol{\beta})\end{array}$ & $\begin{array}{l}\text { Wald } \\
\text { value }\end{array}$ & $\begin{array}{c}\text { P-value } \\
\text { risk }\end{array}$ \\
\hline Sex & -0.107 & 0.972 & 0.324 & 0.899 \\
Age & 0.014 & 7.740 & 0.005 & 1.014 \\
Type of organ metastasis & -1.044 & 22.006 & 0.000 & 0.352 \\
Number of metastatic & -1.617 & 60.020 & 0.000 & 0.199 \\
organs & & & & \\
Treatment methods & 2.680 & 31.409 & 0.000 & 3.547 \\
\hline
\end{tabular}

symptoms were mainly headaches and ostalgia in 139 patients $(25.8 \%)$. The shortest time for patients to see a doctor was 3 days, and the longest was 24 months, with an average of 2.36 months and a median of 2 months. Delayed doctor's visits may be another reason for poor prognosis. ${ }^{1}$

The prognosis of patients with advanced lung cancer was poor, and the 5-year survival rate of patients with stage IV lung cancer was $\sim 1 \%$. In this study, more than half of the patients lived for $>1$ year, but the overall prognosis was poor, with a 3 -year survival rate as low as $1.5 \%$. The prognosis of synchronous metastases was significantly poorer than that of metachronous metastases.

The most common metastatic site of lung cancer was the pleura $(n=112 ; 28.5 \%)$, followed by the brain $(n=100$; $25.4 \%$ ). Patients with single organ metastasis to the bones survived relatively longer, while those with brain metastasis had a similar survival rate, but the survival time of patients with liver metastasis was shorter. The reason for this finding might be that the patients received more timely treatment of bone and brain metastases. If left untreated, the prognosis could be dismal; an effective treatment can improve prognosis and prolong survival. ${ }^{14-16}$

Radiotherapy was the primary treatment, and the effect of chemotherapy on bone and brain metastases was poor. Most patients with bone metastasis experienced severe pain. Thus, the attendance rate and treatment rate were high, and the effect of radiotherapy was beneficial. Treatment was, generally, timely for patients with brain metastasis, as they experienced nervous system symptoms. The curative effect of radiotherapy for single and multiple brain metastases was consistent with the literature. ${ }^{17,18}$ This study found that prognosis was better for patients with single brain metastasis than for those with multiple metastases. In addition, the dose of radiotherapy was positively correlated with survival among patients with brain metastases. Radiotherapy doses of $>50$ Gy predicted better prognosis than did doses of $<50$ Gy. With dose increases, the survival time increased. However, further prospective studies should be conducted to confirm these results.

The prognosis for patients with liver and pleural metastases was poor. Liver metastases often presented as multiple metastases, and the therapeutic effect of both chemotherapy and interventional therapy were poor, identical with that reported in the literature. ${ }^{19,20}$ Radiotherapy may be a good treatment option for single liver metastasis, but was unsuitable for the treatment of multiple liver metastases. In patients with pleural metastasis, malignant pleural effusion was common. Only a small number of patients had well-controlled disease. 
The prognosis was poor for those patients who had extensive pleural metastasis and massive pleural effusion, due to the cachexia resulting from rapid consumption, which was difficult to treat. The prognosis was relatively good for patients without pleural effusion. According to the literature, pleural involvement of lung cancer can be treated surgically, which could improve the survival rate and prolong the survival time. ${ }^{21}$ Therefore, the metastasis of different organs was one of the factors that affected survival.

There was a significant difference in survival time depending on the number of metastatic organs. In this study, there were 393 cases $(72.9 \%)$ of single organ metastasis and 146 cases $(27.1 \%)$ of multiple organ metastases. For patients with single organ, two organs, and three or more organs metastases, the median survival time was 18, 15, and 8 months, respectively. This difference was significant. Therefore, the number of metastatic organs is also a factor that influences survival.

Treatment methods for stage IV lung cancer include chemotherapy, radiotherapy, targeted therapy, and surgery. However, no single treatment is ideal. Because of the impact of traditional concepts, some patients with the first diagnosis of stage IV lung cancer gave up treatment. In this study, 70 patients (13\%) did not accept treatment, which means $>1 / 10$ of patients chose to forego treatment. The median survival time of untreated patients was 5.0 months, compared with 18.0 months for those who received treatment. This difference was significant. As reported in the literature, the median survival time was 3.3 months in patients not receiving chemotherapy, 8.2 months in patients who only received first-line chemotherapy, and 16.2 months in patients who received both first- and second-line chemotherapy. ${ }^{22}$ Therefore, even in patients with advanced lung cancer, survival time can be prolonged after active treatment.

As has been reported elsewhere, different treatment methods also affected survival time. Ma et al reported that in stage IV non-small cell lung cancer, if there were no more than three metastatic organs, the median survival time of concurrent chemotherapy three-dimensional radiotherapy could reach 13.0 months; primary tumor size and dose of radiotherapy were factors affecting overall survival. ${ }^{23}$ Furthermore, prognosis was correlated with Karnofsky Performance Status prior treatment in stage IV non-small cell lung cancer. ${ }^{24,25}$ The prognosis of patients receiving chemotherapy alone was poor, whereas chemotherapy combined with targeted therapy had a better prognosis, indicating that target therapy could improve survival time. ${ }^{26,27}$ Synchronous chemoradiotherapy was important in advanced lung cancer. Radical radiotherapy combined with chemotherapy significantly improves the survival rate and survival time of patients with advanced lung cancer. ${ }^{28,29}$ Hence, treatment methods are another factor that affects survival.

The multivariate Cox regression analysis showed that the survival of patients with stage IV lung cancer was related to age, type of organ metastases, the number of metastatic organs, and treatment methods. Age and treatment methods were positively related to survival.

In conclusion, patients with stage IV lung cancer have various and nonspecific clinical symptoms and experience delayed treatment with poor prognosis. Considering the significant difference between treated and untreated patients, we suggest that patients with advanced stages of disease should still be treated actively, in order to improve survival time. Age, distant organ metastasis, the number of metastatic organs, and treatment methods are the main factors affecting patient survival.

\section{Acknowledgment}

This work was financially supported by Key Research and Development Project of Shandong Province in 2015 (grant no 2015GSF118027).

\section{Disclosure}

The authors report no conflicts of interest in this work.

\section{References}

1. Shao Q, Li J, Li F, et al. Clinical investigation into the initial diagnosis and treatment of 1,168 lung cancer patients. Oncol Lett. 2015;9(2): 563-568.

2. Scheff RJ, Schneider BJ. Non-small-cell lung cancer: treatment of late stage disease: chemotherapeutics and new frontiers. Semin Intervent Radiol. 2013;30(2):191-198.

3. Humphrey LL, Deffebach M, Pappas M, et al. Screening for lung cancer with low-dose computed tomography: a systematic review to update the US Preventive services task force recommendation. Ann Intern Med. 2013;159(6):411-420.

4. Jemal A, Siegel R, Xu J, Ward E. Cancer statistics, 2010. CA Cancer J Clin. 2010;60(5):277-300.

5. Goldstraw P, Crowley J, Chansky K, et al. The IASLC lung cancer staging project: proposals for the revision of the TNM stage groupings in the forthcoming (seventh) edition of the TNM Classification of malignant tumours. J Thorac Oncol. 2007;2(8):706-714.

6. Karam-Hage M, Cinciripini PM, Gritz ER. Tobacco use and cessation for cancer survivors: an overview for clinicians. CA Cancer J Clin. 2014;64(4):272-290

7. Bae J-M, Lee M-S, Shin M-H, Kim D-H, Li Z-M, Ahn Y-O. Cigarette smoking and risk of lung cancer in Korean men: The Seoul Male Cancer Cohort Study. J Korean Med Sci. 2007;22(3):508-512.

8. Sundar IK, Nevid MZ, Friedman AE, Rahman I. Cigarette smoke induces distinct chromatin histone modifications in lung cells: implication in pathogenesis of COPD and lung cancer. J Proteome Res. 2014;13(2):982-996. 
9. Hesterberg TW, Long CM, Bunn WB, et al. Health effects research and regulation of diesel exhaust: an historical overview focused on lung cancer risk. Inhal Toxicol. 2012;24(Suppl 1):1-45.

10. Sprague BL, Trentham-Dietz A, Klein BE, et al. Physical activity, white blood cell count, and lung cancer risk in a prospective cohort study. Cancer Epidemiol Biomarkers Prev. 2008;17(10):2714-2722.

11. Eberth JM. Lung cancer screening with low-dose CT in the United States. J Am Coll Radiol. 2015;12(12):1395-1402.

12. Sharma D, Newman TG, Aronow WS. Lung cancer screening: history, current perspectives, and future directions. Arch Med Sci. 2015;11(5): 1033-1043.

13. Deffebach ME, Humphrey L. Lung cancer screening. Surg Clin North Am. 2015;95(5):967-978.

14. Caffo M, Barresi V, Caruso G, et al. Innovative therapeutic strategies in the treatment of brain metastases. Int J Mol Sci. 2013;14(1): 2135-2174.

15. Antoni D, Noël G, Mornex F. The role of whole brain radiation therapy for brain metastases. Bull Cancer. 2013;100:15-22.

16. Sugiura H, Yamada K, Sugiura T, Hida T, Mitsudomi T. Predictors of survival in patients with bone metastasis of lung cancer. Clin Orthop Relat Res. 2008;466(3):729-736.

17. Tsao MN, Rades D, Wirth A, et al. Radiotherapeutic and surgical management for newly diagnosed brain metastasis(es): an American Society for Radiation Oncology evidence-based guideline. Pract Radiat Oncol. 2012;2(3):210-225.

18. Tamari K, Suzuki O, Hashimoto N, et al. Treatment outcomes using CyberKnife for brain metastases from lung cancer. J Radiation Res. 2015;56(1):151-158.

19. Castañón E, Rolfo C, Viñal D, et al. Impact of epidermal growth factor receptor (EGFR) activating mutations and their targeted treatment in the prognosis of stage IV non-small cell lung cancer (NSCLC) patients harboring liver metastasis. J Transl Med. 2015;13:257.

20. Wu KL, Tsai MJ, Yang CJ, et al. Liver metastasis predicts poorer prognosis in stage IV lung adenocarcinoma patients receiving first-line gefitinib. Lung Cancer. 2015;88(2):187-194.
21. Iida T, Shiba M, Yoshino I, Miyaoka E, et al; Japanese joint committee of lung cancer registry. Surgical intervention for non-small-cell lung cancer patients with pleural carcinomatosis: results from the Japanese lung cancer registry in 2004. J Thorac Oncol. 2015;10(7):1076-1082.

22. Barton MK. Patients of all ages with advanced non-small cell lung cancer are not receiving chemotherapy. CA Cancer J Clin. 2015;65(5): $337-338$.

23. Zhu M, Bing L, Tao L, et al. A prospective, multicenter, phase II clinical study of three-dimensional radiotherapy with concurrent chemotherapy for stage IV non-small-cell lung cancer - PPRA-RTOG003. Chinese J Rad Oncol. 2015;24(4):359-364.

24. Lopez-Guerra JL, Gomez D, Zhuang Y, et al. Prognostic impact of radiation therapy to the primary tumor in patients with non-small cell lung cancer and oligometastasis at diagnosis. Int J Radiat Oncol Biol Phys. 2012;84(1):61-67.

25. Parikh RB, Cronin AM, Kozono DE, et al. Definitive primary therapy in patients presenting with oligometastatic non-small cell lung cancer. Int J Radiat Oncol Biol Phys. 2014;89(4):880-887.

26. Li XN, Qiu D, Pan X, Hou XX. Mutation of the epidermal growth factor receptor gene and its impact on the efficacy of gefitinib in advanced non-small cell lung cance. Int J Clin Exp Med. 2015;8(4):5397-5405.

27. Kaichun L, Jiayan C. Treatment options in the elderly patients with advanced non-small-cell lung cancer. Chinese J Oncol. 2015;37(10): 721-724. Chinese.

28. McCloskey P1, Balduyck B, Van Schil PE, Faivre-Finn C, O’Brien M Radical treatment of non-small cell lung cancer during the last 5 years. Eur J Cancer. 2013;49(7):1555-1564.

29. Lv Jiahua, Li Tao, Liu Li, et al. A prospective phase II study of consolidation chemotherapy after concurrent chemoradiotherapy for oligometastatic stage IV non-small cell lung cancer. Chinese J Radiat Oncol. 2015;24(6):611-614.
OncoTargets and Therapy

\section{Publish your work in this journal}

OncoTargets and Therapy is an international, peer-reviewed, open access journal focusing on the pathological basis of all cancers, potential targets for therapy and treatment protocols employed to improve the management of cancer patients. The journal also focuses on the impact of management programs and new therapeutic agents and protocols on

\section{Dovepress}

patient perspectives such as quality of life, adherence and satisfaction. The manuscript management system is completely online and includes a very quick and fair peer-review system, which is all easy to use. Visit http://www.dovepress.com/testimonials.php to read real quotes from published authors. 